\title{
Status of the Mid-IR ELT Imager and Spectrograph (METIS)
}

\author{
Bernhard R. Brandl*a,b ${ }^{\text {a }}$ Olivier Absil ${ }^{\mathrm{c}}$, Tibor Agócs ${ }^{\mathrm{d}}$, Nicola Baccichet ${ }^{\mathrm{e}}$, Thomas Bertram ${ }^{\mathrm{f}}$, Felix \\ Bettonvil $^{\mathrm{a}, \mathrm{d}}$, Roy van Boekel ${ }^{\mathrm{f}}$, Leonard Burtscher ${ }^{\mathrm{a}, \mathrm{d}}$, Ewine van Dishoeck ${ }^{\mathrm{a}}$, Markus Feldt ${ }^{\mathrm{f}}$, Paulo J. V. \\ Garcia $^{\mathrm{g}}$, Alistair Glasse ${ }^{\mathrm{h}}$, Adrian Glauser ${ }^{\mathrm{i}}$, Manuel Guedel ${ }^{\mathrm{j}}$, Christoph Haupt ${ }^{\mathrm{l}}$, Matthew A. \\ Kenworthy ${ }^{\mathrm{a}}$, Lucas Labadie ${ }^{\mathrm{e}}$, Werner Laun ${ }^{\mathrm{f}}$, Dirk Lesman ${ }^{\mathrm{d}}$, Eric Pantin ${ }^{\mathrm{k}}$, Sascha P. Quanz ${ }^{\mathrm{i}}$, Ignas \\ Snellen ${ }^{\mathrm{a}}$, Ralf Siebenmorgen ${ }^{1}$, Hans van Winckel ${ }^{\mathrm{m}} \&$ the METIS Team
}

a'Leiden Observatory, Leiden University, P.O. Box 9513, 2300 RA Leiden, The Netherlands; ${ }^{b}$ Faculty of Aerospace Engineering, Delft University of Technology, Kluyverweg 1, 2629 HS Delft, The Netherlands;

${ }^{c}$ Université de Liège, STAR Institute, 19c allée du Six Août, B-4000 Liège, Belgium;

dNOVA O/IR Group, P.O. Box 2, 7990 AA Dwingeloo, The Netherlands;

eI. Physikalisches Institut, Universität zu Köln, Zülpicher Straße 77, 50937 Köln, Germany;

${ }^{f}$ Max-Planck-Institut für Astronomie, Königstuhl 17, 69117 Heidelberg, Germany;

'Universidade do Porto, Faculdade de Engenharia, CENTRA research unit - SIM group, Rua Dr.

Roberto Frias, 4200-465 Porto, Portugal

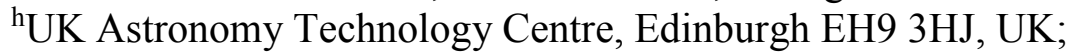

iSwiss Federal Institute of Technology (ETH Zürich), Department of Physics, Institute for Particle

Physics and Astrophysics, Wolfgang-Pauli-Strasse 27, CH-8093 Zürich, Switzerland;

jUniversity of Vienna, Department of Astrophysics, Türkenschanzstrasse 17, A-1180 Vienna, Austria;

${ }^{\text {k} C o m m i s s a r i a t ~ a ̀ ~ l ' E n e r g i e ~ A t o m i q u e, ~ I n s t i t u t ~ d e ~ R e c h e r c h e ~ s u r ~ l e s ~ L o i s ~ F o n d a m e n t a l e s ~ d e ~ l ' U n i v e r s, ~}$ Service d'Astrophysique, Orme des Merisiers, 91191 Gif sur Yvette, France;

${ }^{1}$ European Southern Observatory, Karl-Schwarzschild-Str. 2, 85748 Garching bei München, Germany;

mInstituut voor Sterrenkunde, K.U.Leuven, Celestijnenlaan 200D, B-3001 Leuven, Belgium.

\begin{abstract}
METIS is one of the first three scientific instruments on the ELT, expected to see first light in 2025. METIS will provide diffraction limited imaging, coronagraphy, and low resolution slit spectroscopy over the $3-19 \mu \mathrm{m}$ range. Furthermore, METIS will provide a unique combination of high resolution $(R \sim 100,000)$ integral field spectroscopy and coronagraphy from $2.9-5.3 \mu \mathrm{m}$. The latter can be reconfigured to obtain a wider instantaneous wavelength coverage in exchange for integral field coverage. While METIS is a multi-purpose instrument for a wide range of infrared astronomy, its greatest strengths will be in the studies of exoplanets and proto-planetary disks.

After two years into the preliminary design phase, the design of METIS has reached an advanced stage. This paper provides an update on the areas which have most advanced in the recent past: the main science drivers, the optical and opto-mechanical design, adaptive optics and the coronagraphy concept. We also describe the calibration of the instrument, give an overview of its observation modes, and present the schedule toward first light.
\end{abstract}

Keywords: METIS, ELT, mid-infrared, cryogenic, preliminary design, science, calibration, adaptive optics

*brandl@strw.leidenuniv.nl; phone +31 715275830

Ground-based and Airborne Instrumentation for Astronomy VII, edited by Christopher J. Evans, Luc Simard, Hideki Takami, Proc. of SPIE Vol. 10702, 107021U · C 2018 SPIE · CCC code: 0277-786X/18/\$18 · doi: 10.1117/12.2311492 


\section{INTRODUCTION}

The 'Mid-infrared ELT Imager and Spectrograph' (METIS) is one of the first three scientific instruments on ESO's Extremely Large Telescope (ELT). METIS will provide the following scientific observing capabilities:

- Imaging at $3-19 \mu \mathrm{m}$. The imager module includes:

○ low resolution (few hundreds) long-slit spectroscopy,

$\circ$ coronagraphy for high contrast imaging.

- High resolution $(\mathrm{R} \sim 100,000$ ), integral field (IFU) spectroscopy at $3-5 \mu \mathrm{m}$. The IFU spectroscopy module includes:

- a mode with extended instantaneous wavelength coverage ( 300 $\mathrm{nm}$ instead of $50 \mathrm{~nm})$,

- a combined IFU+coronagraphy mode.

All observing modes work at the diffraction limit of the 39m ELT with a single conjugate AO (SCAO) system, which yields an angular resolution of 23 milliarcseconds at $3.5 \mu \mathrm{m}$ wavelength. At this wavelength, METIS will have a point source sensitivity (10- $\sigma$ in 1 hour) of $21.2 \mathrm{mag}$ (or about $1 \mu \mathrm{Jy}$ ). Focusing mostly on compact targets, the METIS field of view is relatively small: $\sim 10^{\prime \prime} \times 10^{\prime \prime}$ in imaging and $\sim 1.0^{\prime \prime} \times 0.5^{\prime \prime}$ in IFU spectroscopy mode at L- and M-bands.

METIS is being built by an international consortium with the following members: NOVA (The Netherlands, PI: Bernhard Brandl), MPIA Heidelberg (Germany), CEA-Saclay (France), UKATC (United Kingdom), KU Leuven (Belgium), ETH Zürich (Switzerland), A* Consortium (Austria), Centra (Portugal), and the University of Cologne (Germany).

Since METIS will undergo its preliminary design review in May 2019, the technical information presented in this paper must be considered preliminary and is subject to change. Additional information can also be found in previous on METIS papers at this SPIE conference series [1], [2].

\section{SCIENCE CASE}

METIS is going to be a multi-purpose instrument, attractive to a wide scientific community, and able to make significant contributions to a variety of research fields. Star formation and stellar cluster research, evolved stars, AGNs and local star bursts, as well as Solar System science, will benefit from the unique capabilities of METIS, combining high-spatial resolution and high-spectral resolution with unprecedented sensitivity for a ground-based mid-infrared instrument. In the past few years, however, it became clear that two areas - circumstellar disk science and extrasolar planet research - are driving the instrument! Therefore, we provide a few concrete examples for these two areas below. A more comprehensive and quantitative description of the METIS science case is currently underway, and will be available in spring 2019.

\subsection{Circumstellar disks and planet formation}

Literally all available METIS observing modes will be employed to study and investigate the structure (morphological and kinematic), composition, and evolution of circumstellar disks. For instance, gas-poor debris disks and exozodiacal dust in nearby stellar systems will primarily be studied with (high-contrast) imaging modes with the aim to constrain the extent and brightness of these dust belts and potentially link them to planets orbiting in these systems. Young, gas-rich disks will be prime targets for observations with the L and M band IFU to study the dynamics of the molecular gas and do kinematic imaging of the gas in the planet-forming regions. In addition, the IFU will provide the possibility to search directly for kinematic imprints of forming gas giant planets and their suspected circumplanetary disks, which could then potentially be imaged in broad-band continuum.

Indirect signs of forming planets, such as gaps and cavities in circumstellar disks, can also be studied with METIS. It is the combination of images at near-infrared, mid-infrared and sub-millimeter wavelengths, mapping the relative spatial distribution of dust grains of different size, that is needed to distinguish between different disk-sculpting mechanisms [3], [4]. In this context it is important to recall that METIS in the N-band $(\sim 10 \mu \mathrm{m})$ will provide the same spatial resolution as current NIR instruments installed at 8-m telescopes studying disks in scattered light, such as VLT/SPHERE or Gemini/GPI. In Figure 1 we show simulated METIS N-band and ALMA $850 \mu \mathrm{m}$ images of a disk with embedded 
planets of varying mass. The images result from a full hydrodynamic calculation, including a prescription for dust evolution and dynamics [5]. As shown in this study, the separation between the micron- and mm-sized dust grains is very sensitive to the mass of the embedded planet.

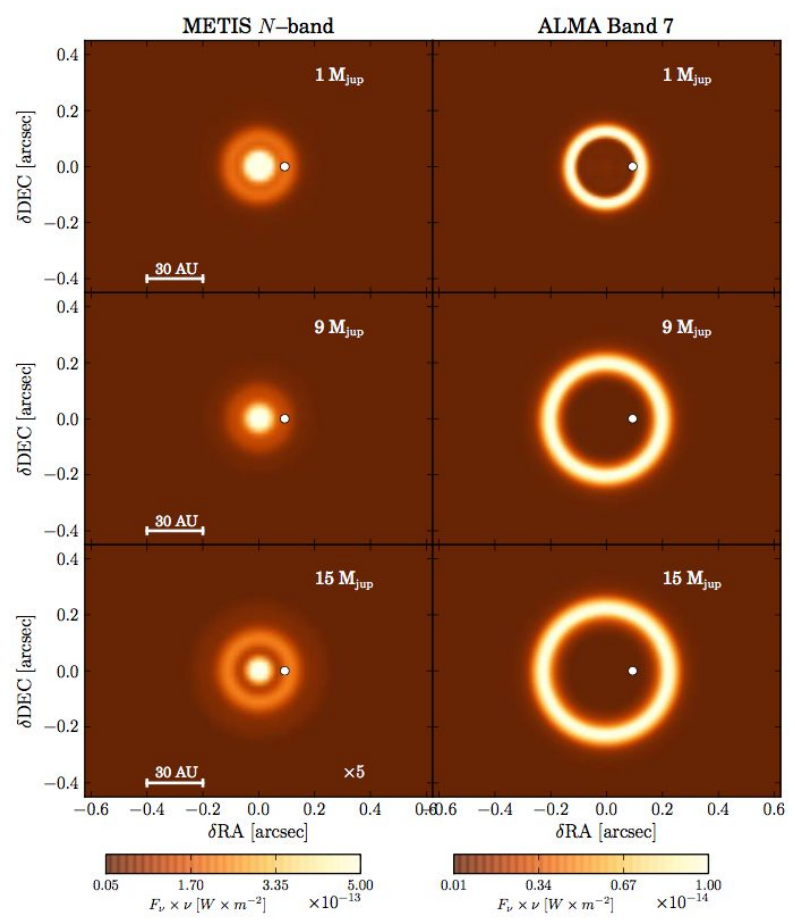

Figure 1 Simulated images of disk-planet interaction models for the case of an embedded planet orbiting at $13 \mathrm{AU}$ (white dot) around a solar-mass star. The disk is assumed to be at a distance of 150 parsecs (pc). The left and right columns correspond to METIS images at N-band and ALMA images at $850 \mu \mathrm{m}$, respectively. Top, middle and bottom rows show images obtained for planet masses of 1,9 and 15 MJupiter, respectively. The images were obtained following the procedure of [5].

Finally, METIS will also be able to investigate the chemical composition of protoplanetary disk material by studying water and organics in the innermost disk regions and trying to image water ice frozen onto dust grains in cool enough regions. PAHs and related carbonaceous species can be studied via imaging and spectroscopy in the L and $\mathrm{N}$ band [6] and potentially the question when and where the crystallization of silicates takes place can be addressed.

\subsection{Exoplanet detection and characterization}

Extremely large telescopes present incredible opportunities to discover and characterize diverse planetary systems, even potentially habitable worlds, and in particular AO-assisted (high-contrast) imaging and (high-resolution) spectroscopy in the $3-10 \mu \mathrm{m}$ range are powerful tools to detect and study exoplanets. METIS will be superior to JWST for high contrast imaging at the innermost circumstellar regions in the thermal-IR, and complementary to high contrast observations at shorter wavelengths, in space or with second- or third-generation extreme AO ELT instruments.

METIS will combine high-spectral resolution spectroscopy with coronagraphic imaging [7] allowing for the detection and spectroscopic characterization of a variety of planet types, from transiting and non-transiting Hot-Jupiters to Neptune-like objects and potentially even super-Earths. Spin rates can be measured [8], [9], and compared to predictions for planet formation models and the spin rates observed in our Solar System. This technique also allows for Doppler Imaging to map the atmospheres of (giant) planets in two dimensions providing information about potential cloud coverage [10]. 
In addition, measuring exoplanet atmospheric isotope ratios could become a prime science case for METIS [11]. These ratios provide unique insights in the formation and evolution of planets and their atmospheres, in particular the $\mathrm{D} / \mathrm{H}$ ratio. HDO is best targeted at $3.7 \mu \mathrm{m}$. Counter-intuitively, HDO is easier to detect in cooler planets because it is less shielded by water absorption. We estimate that if the atmosphere of Proxima $b$ is rich in water, this isotopologue could be detected in $\sim 1$ night of observing time in its reflected-light spectrum [11].

Concerning "classical" high-contrast imaging, Quanz et al. [12] already showed that - depending on the final contrast performance - METIS might be able to take a direct picture of a few (small) planets around the nearest stars. Specifically, searching for terrestrial planets in the habitable zone of the $\alpha$ Centauri system in the $\mathrm{N}$ band will be a "high risk - high reward" science case, unique to an instrument like METIS.

In addition, METIS will be able to image dozens of planets that were previously detected via radial velocity (RV) searches or with GAIA [13] via astrometry and have an empirical estimate for the mass (Figure 2). Until today, not a single exoplanet is known, of which both the mass and the luminosity have been directly measured. Also here, METIS will truly open up a new line of exoplanet research.
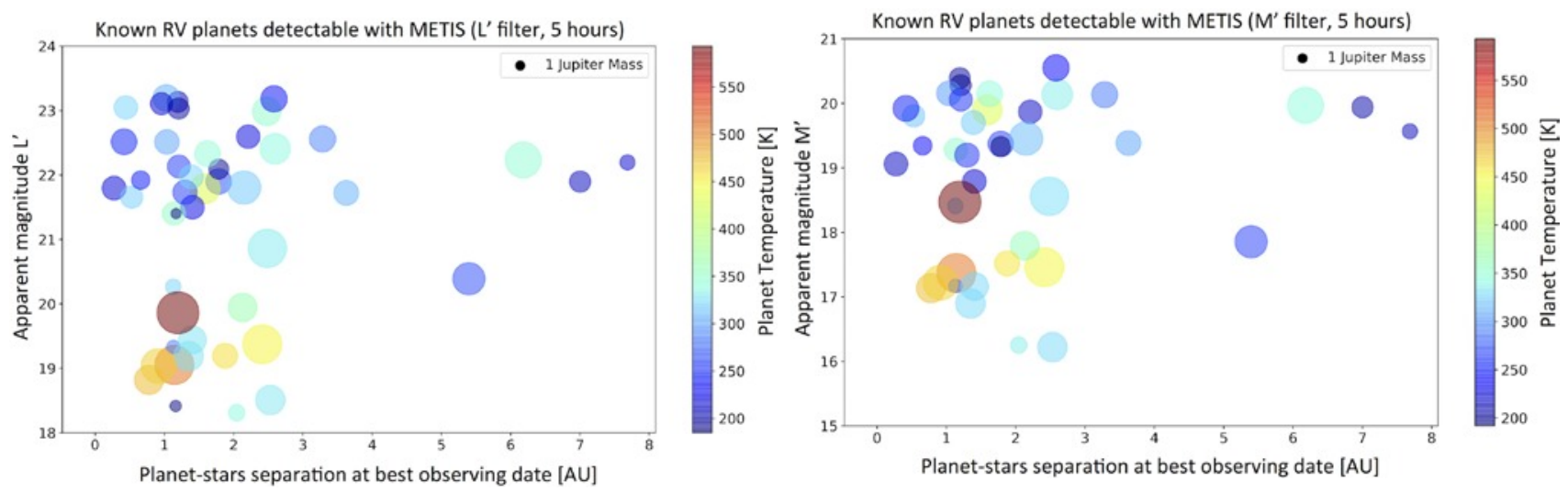

Figure 2 Updated estimates on the number and properties of known exoplanets detected via radial velocity searches [14] that can - very likely - be directly imaged with METIS. Both plots assume background-limited performance starting at $5 \lambda / D$ and 5 hours on-source integration time. The results for the $L^{\prime}$ filter are shown on the left and for $M^{\prime}$ on the right. Planet temperatures are estimated using evolutionary models and are only lower limits as stellar insolation is not yet taken into account (i.e., the planets could be hotter and even easier to detect). Even within 1 hour of integration time, the number of detectable planets is larger than 20, and many more targets will become available in the coming years with the continuation of long-term RV programs and the results from the GAIA satellite.

\section{OVERVIEW OF THE OPTICAL SYSTEM}

The optical overview of METIS is shown in Figure 3. METIS consists of two diffraction-limited imagers operating in the L/M (IMG-LM) and N/Q (IMG-NQ) bands, and an Integral Field Unit (IFU)-fed, diffraction-limited, high-resolution $(\mathrm{R}=100,000) \mathrm{LM}$ band Spectrograph (LMS). It also provides coronagraphy at focal and pupil plane for all operating modes for high contrast imaging. The Common Fore Optics (CFO) provides the opto-mechanical interfaces between the various subsystems and is responsible for chopping, image de-rotation, pupil stabilization, thermal background and stray light reduction [15].

All the mentioned subsystems, in conjunction with an infrared (HK bands) wavefront sensor of the single conjugate Adaptive Optics (SCAO, here: SCA) system, are located in the cryostat. The Warm Calibration Unit (WCU, section 7) is responsible for routine daily daytime calibrations and alignment verification of METIS during the assembly, integration and verification (AIV) phase. The METIS cryostat and the WCU are held in position by the Warm Support Structure (WSS) [16]. 
The optical design of METIS is driven by the requirement that that the quality and alignment of the optical components within METIS shall provide diffraction limited (Strehl $\geq 80 \%$ ) performance at $\lambda>3 \mu \mathrm{m}$ in all modes over the entire field of view (FOV). The static WFE budget, corresponding WFE modelling and verification approach (based on Power Spectral Density functions) are discussed in [17].

The optical interface between the telescope and METIS is the ELT focal plane, which is located $1000 \mathrm{~mm}$ upstream of the METIS cryostat window. The ZnSe window is slightly tilted (facing down) in order to avoid contamination on the surface during operation. It is wedged to compensate for the dispersion introduced by the window itself and avoid any Fabry-Perot fringing. Just outside the cryostat the retractable Warm Calibration Unit (WCU) feed mirror is located.

Light entering into the cryostat first propagates through the CFO. Its optical design is based on a double re-imager configuration, both re-imagers work as 1:1 relays. The CFO is an all-spherical system that consists of a classical and a re-optimized Offner relay, providing very high Strehl ratio for the IMG and LMS science subsystems. Two focal and pupil planes provided by the double relay CFO design make it possible to accommodate various High Contrast Imaging (HCI) modes and reduce stray light significantly. The AO pick-off, located after the derotator and the pupil stabilization mirror, directs the near infrared light ( $\mathrm{H}$ and $\mathrm{K}$ bands) into the SCA subsystem. In the second pupil of the CFO, a 2Dsteerable mirror (the beam chopper) is positioned, which switches the beam between the target and a nearby reference sky ( $\leq 5^{\prime \prime}$ in any direction) during observation for the characterization of the variable thermal background.

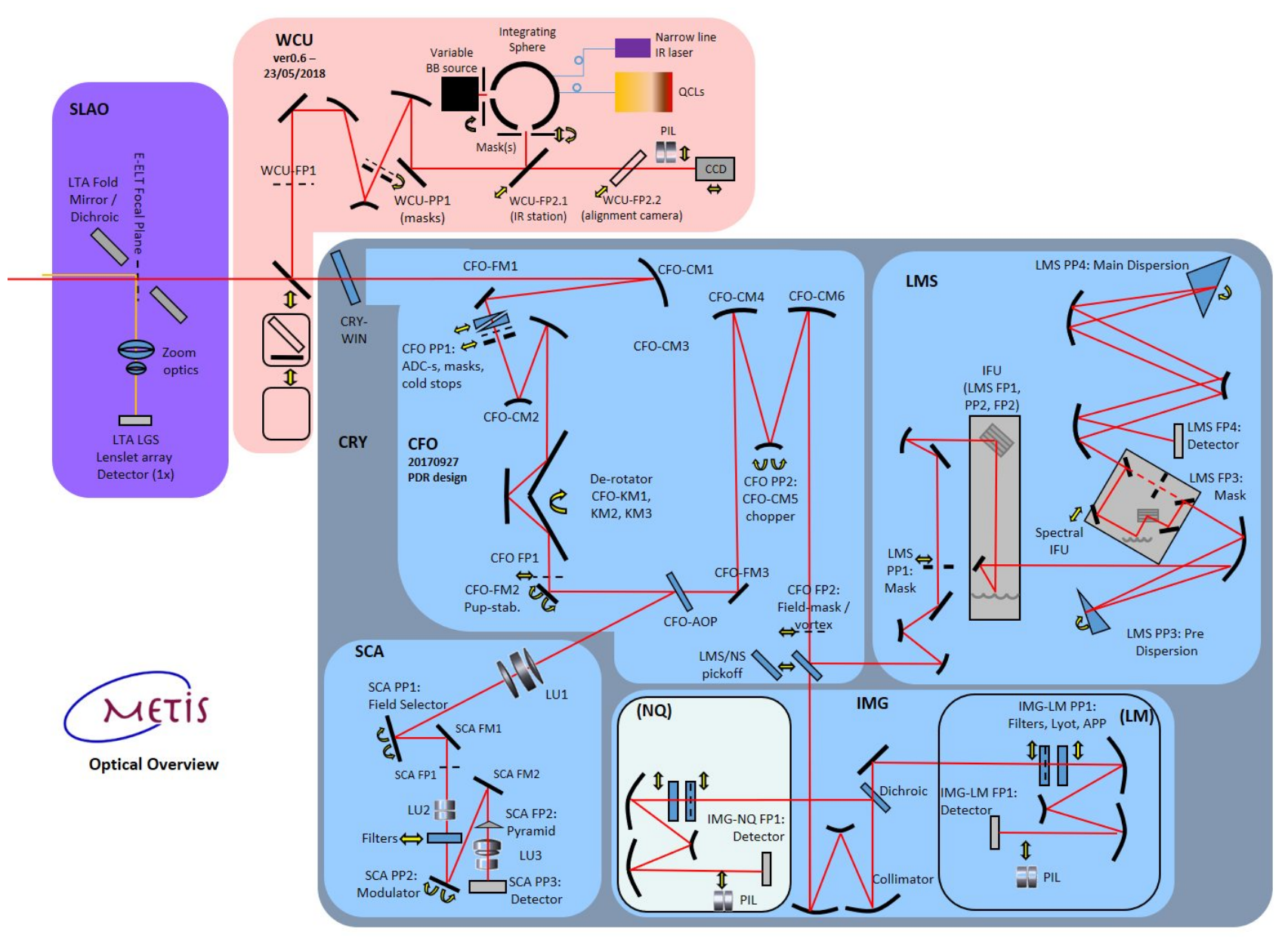

Figure 3 The optical overview of METIS. For details see main text. 
The SCA subsystem is based on a Pyramid Wave Front Sensor (WFS). A three lens achromat reimages the pupil plane, where the field selector is located, which is able to select the target object anywhere within the complete FOV (27" across). Next, another doublet reimages the pupil plane, where a very fast tip-tilt mirror is located, that modulates the PSF over the four sides of "the pyramid", the key component of the SCAO wavefront sensor. The pyramid is a refractive component splitting the light into four beams, and after a last achromatic triplet, four separate pupils are formed on the WFS focal plane detector, a Saphira from Leonardo. The four pupil images are then used to retrieve the phase information to control M4/M5 of the ELT accordingly.

The second focal plane (FP2) of the CFO constitutes also the optical interface between the CFO and the science subsystems of the instrument. After FP2, the LMS pick-off reflects the light towards the high-resolution spectrograph. When the pickoff is retracted, the full field of view is transmitted towards the L/M and N/Q band Imagers (IMG).

The high resolution $(\mathrm{R}=100,000$ at $4.65 \mu \mathrm{m}) \mathrm{LMS}$ is an integral field spectrometer, which operates in the $2.9-5.3 \mu \mathrm{m}$ wavelength range. After the LMS pickoff, the transfer optics reimages the focal plane onto the slicing mirrors of the IFU, which reformats the image. The input FOV is slightly larger than 0.5 square arcseconds, and the slice width is matched to the diffraction limit, $\lambda / \mathrm{D}$, at $\lambda=3.7 \mu \mathrm{m}$, which corresponds to 19.6 milliarcseconds on the sky. The pseudo-slit provided by the IFU is the input optical interface for the main optical unit of the LMS, which is based on an Echelle spectrometer. The main disperser is a silicon-based, immersed grating [18], where the diffraction occurs inside the material. Regarding the optical design of the LMS, the immersed grating is positioned in a near-Littrow configuration inside a double-pass, three mirror anastigmat (TMA), and the necessary order separation is achieved by a ZnSe predisperser and a spatial filter. A so-called "spectral IFU"-unit, which follows similar design principles as an IFU to provide an alternative to cross-dispersion, can achieve extended wavelength coverage [19]. The LMS records the spectra using a $2 \times 2$ mosaic of HAWAII-2RG detectors.

The IMG provides diffraction-limited imaging over a FOV of $10^{\prime \prime} \times 10^{\prime \prime}$ at $\mathrm{L}, \mathrm{M}$ and N, Q bands. In addition to plain imaging, it provides coronagraphy and long slit spectroscopy $(450 \leq \mathrm{R} \leq 2000)$ at $\mathrm{L}, \mathrm{M}$ and $\mathrm{N}$ bands. The optical system of the IMG uses a common TMA to collimate the beam, and subsequently, a dichroic splits the light into the LM and NQ bands. Since the dichroic is upstream of the last pupil, we use the pupil plane in each imager module to insert various spectral filters, coronagraphic masks, and grism dispersers. The L/M and the N/Q band cameras are also TMAs, which provide diffraction-limited image quality at their specific wavelength range. The IMG provides a pixel scale of 5.2 milliarcseconds/pixel at L/M-band, and 10.8 milliarcseconds/pixel at N/Q-band. The pixel sampling corresponds to the FOV of $10^{\prime \prime} \times 10^{\prime \prime}$ at L/M and N/Q bands, assuming one $2048 \times 2048$ pixel Teledyne HAWAII-2RG and one $1024 \times 1024$ pixel Raytheon Aquarius focal plane detector for the L/M and N/Q arms, respectively. The IMG also contains pupil imaging options in both channels, which are realized by inserting one ZnSe-lens in front of the TMA and one behind. The front-lens is mounted in one of the pupil-wheels, while the rear lens is moved by a dedicated mechanism.

The realization and folding of the optical system within the cryostat is shown in Figure 4 (left).

\section{OPTO-MECHANICAL SYSTEM}

\subsection{Packaging concept}

A modular approach was used for the mechanical layout of METIS (Figure 4). The cryostat, cold central structure, and the CFO together form the heart of the instrument, to which all other sub-systems are attached. The cold central structure provides the mechanical and thermal interfaces to all cryogenically cooled sub-systems, as well as the thermal interface to the pulse tube coolers for the steady state cooling (section 5). The cold central structure is also equipped with the $\mathrm{LN}_{2}$ pre-cooling system and cold cable harnesses. The LMS, IMG, and SCA are mounted to the sides and bottom of the cold central structure (Figure 4).

\subsection{The Cryostat}

A large cryostat of $3 \mathrm{~m}$ in diameter and $4 \mathrm{~m}$ in height is used to cool the cold instrument. The cryostat consists of four segments, of which the top section is the stiffest and most complex segment (Figure 5, left). This segment provides the interfaces between the cold central structure, the hexapod of the warm support structure, and the warm calibration unit on top. It also hosts the entrance window, the vacuum pumps, the pulse tube coolers, and is equipped with the feedthroughs 
for the cables, which are routed over the cold central structure. The other cryostat components are the remaining cryostat wall segments, the multi-layer insulation (MLI), and the $\mathrm{LN}_{2}$ radiation shield (Figure 5, left). These components can be mounted and dismounted one by one. The height of the wall segments is the same to minimize the space required during integration.
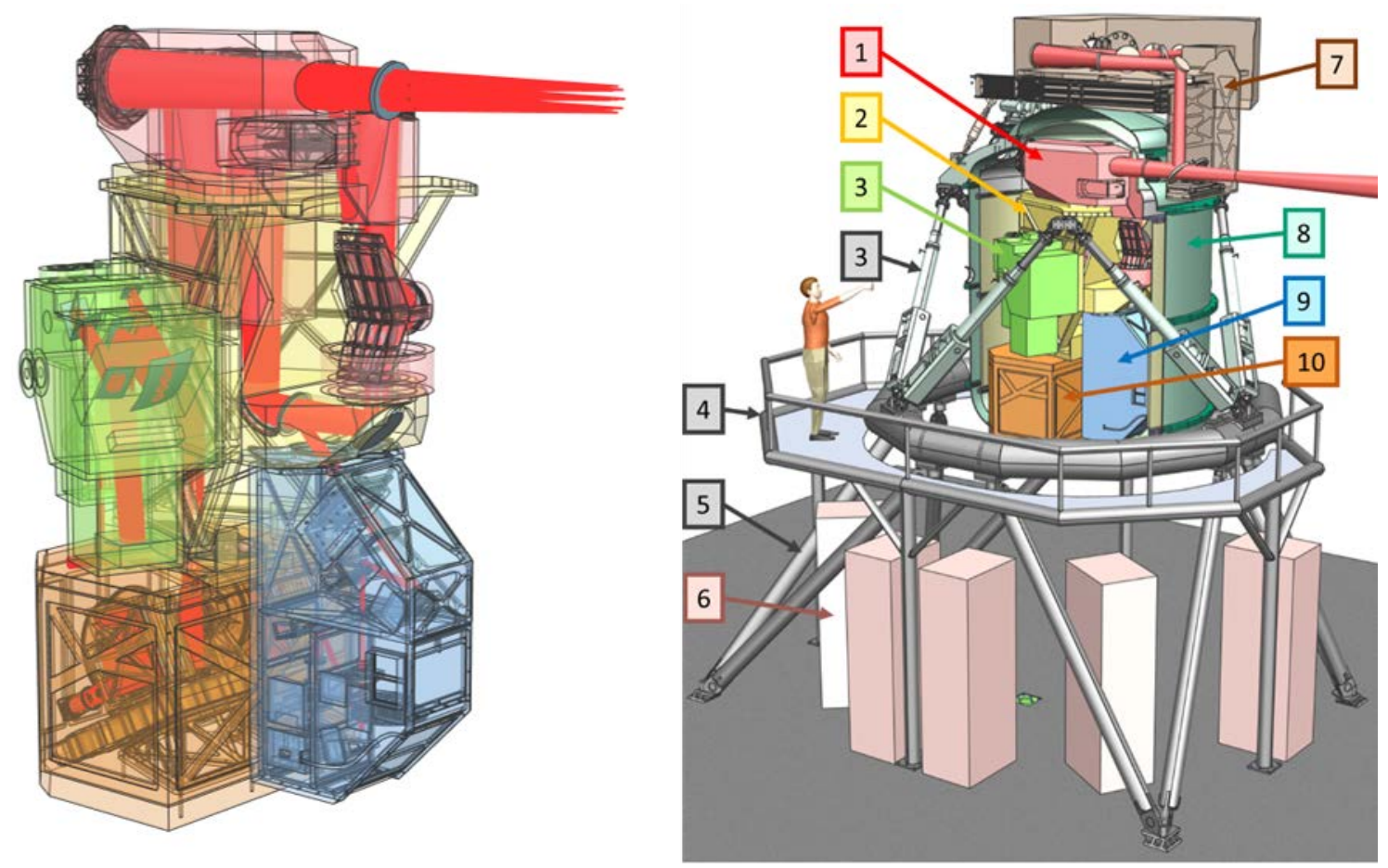

Figure 4 Left: The incorporation and folding of the optical system within the cryostat. From top to bottom: the CFO (pink), cold central structure (yellow), LMS (green), SCA (blue), and IMG (brown). Right: the packaging of the various instrument modules within the cryostat: 1 - common fore optics (CFO), 2 - cold central structure, 3 (black) - motorized hexapod, 4 - access platform, 5 - elevation platform, $(3,4$, and 5 together form the warm support structure, WSS), 6 - electronics racks, 7 - warm calibration unit (WCU), 8 - cryostat, 9 - SCA, 10 IMG.

The cryostat is supported by the warm support structure, which consists of three main components:

- Motorized hexapod - interfaces to the cryostat and is used for the rough alignment between METIS and the ELT.

- Elevation platform - supports the hexapod and attaches to the main interfaces points of the Nasmyth platform.

- Access platform - allows access to parts of the instrument and supports cable trays to the top of the cryostat.

Most electronics cabinets are placed below the warm support structure. However, due to the generally large distances at the ELT, at least one cabinet will be placed close to the cryostat to serve those components for which short cable lengths are critical, e.g. detectors and encoders.

\section{CRYOGENIC CONCEPT}

The METIS cryostat needs to provide three temperature zones with given maximum temperatures (which may actually be lower), which fulfil the thermal requirements of each scientific subsystem (Figure 5, right).

The first temperature level is at about $70 \mathrm{~K}$. It includes the CFO, the LMS, the SCA, and LM-band imager (IMG). All of these units are mounted to the central cold structure, which is cooled by the first stages of three pulse tube coolers. 

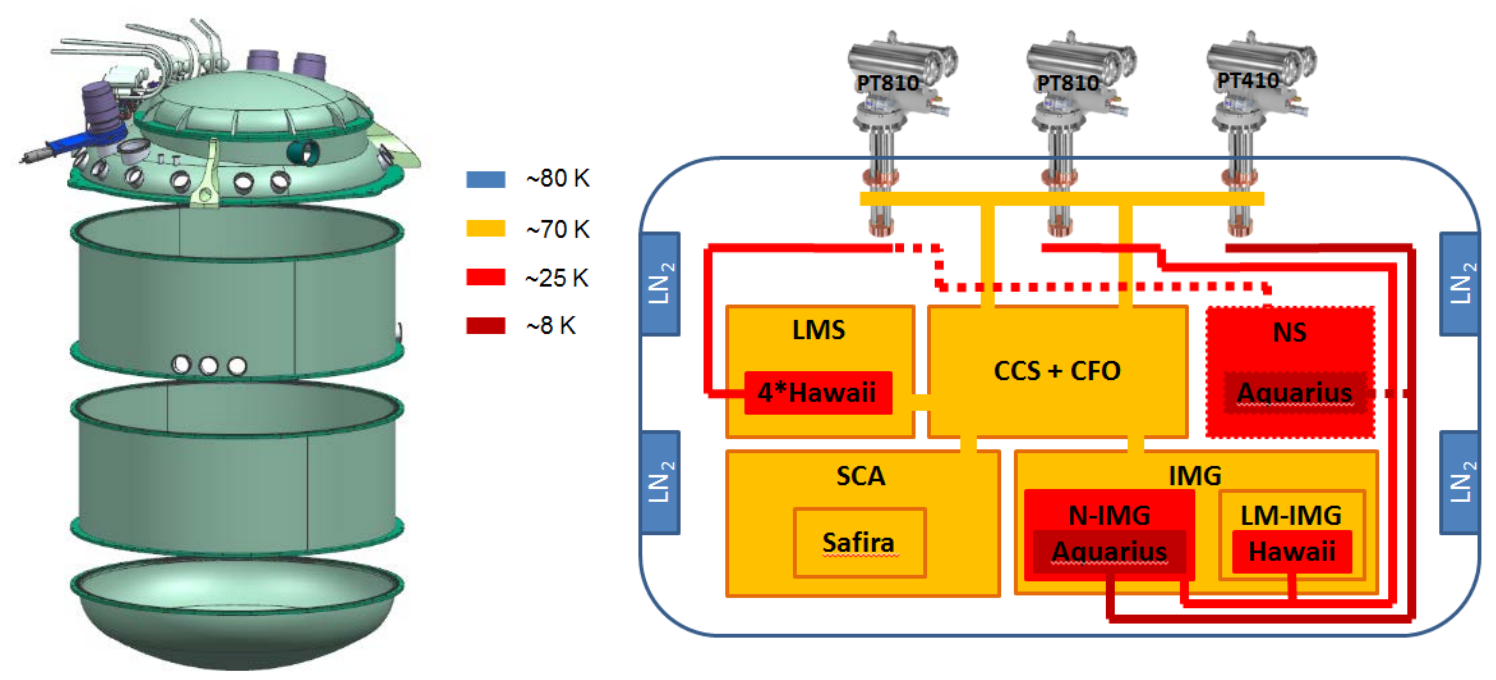

Figure 5 Left: the main components of the cryostat. Right: schematic overview of the METIS cooling concept.

The second temperature level is at about $25 \mathrm{~K}$. This is the temperature range required by the HAWAII-2RG detectors and the optics of the N-band imager module. Here we use the second stages of two pulse-tube coolers (e.g., PT810 from CRYOMECH). The METIS concept also contains the provisions for an optional high-resolution N-band spectrograph, which would also require about $25 \mathrm{~K}$ for its opto-mechanical system. The cooling power provided by the pulse tube coolers in this temperature range would be sufficient to also cool the optional N-band spectrograph.

The coldest temperature requirement, namely $8 \mathrm{~K}$, comes from the Aquarius (Si:As BIB) detector of the N-band imager. This temperature is provided by the second stage of the pulse tube cooler (e.g., the PT410). A possibly additional Aquarius of the optional N-band spectrograph could also be cooled by this cooler.

The complete cold structure is built into a radiation shield, which is cooled by $\mathrm{LN}_{2}$. In order to simplify assembly and integration, the radiation shield is segmented in the same way the vacuum vessel is segmented. The individual parts of the vacuum vessel will be permanently connected to the corresponding parts of the radiation shield. Each segment of the radiation shield will have its own $\mathrm{LN}_{2}$ vessel. A precooling system will cool the cold structure by a flow of $\mathrm{LN}_{2}$ (Figure 5 , right).

\section{ADAPTIVE OPTICS}

All observing modes of METIS work at the diffraction-limit and therefore require adaptive optics (AO) to provide the necessary temporal and spatial correction of the incoming wavefront. METIS will be equipped with a classical singleconjugate AO (SCAO) system, well suited for the high contrast science cases. The feasibility and performance of an additional laser guide star AO system for increased sky coverage is currently under study.

The SCAO system consists of a number of entities, distributed across the instrument and telescope domain. Its control scheme is outlined in Figure 6. An SCAO module with a NIR Pyramid wavefront sensor measures the wavefront aberrations. The Real-Time Computer (RTC) processes the wavefront sensor data and calculates correction signals, which are sent via the Central Control System (CCS) of the ELT to the main actuators of the wavefront control: the ELT provides the deformable mirror M4, with a total of 5316 actuators, and the field stabilization mirror M5. The frequency of the wavefront control loop will be up to $1 \mathrm{kHz}$.

Like most other subsystems of METIS, the SCAO module resides inside the cryostat. As a consequence, actuators such as the field selector and the modulator have to operate at a temperature of $70 \mathrm{~K}$. A filter wheel allows to select bandpass and neutral density filters to avoid saturation on the brightest targets. The wavefront sensor signal will be obtained with a Leonardo Saphira APD detector. 
The AO control system is, as part of the Instrument Control System of METIS, responsible for the AO wavefront correction and a number of related secondary control tasks, such as pupil stabilization and closed loop compensation for non-common path aberration for high contrast imaging observations. More details on the METIS AO system can be found in [20].

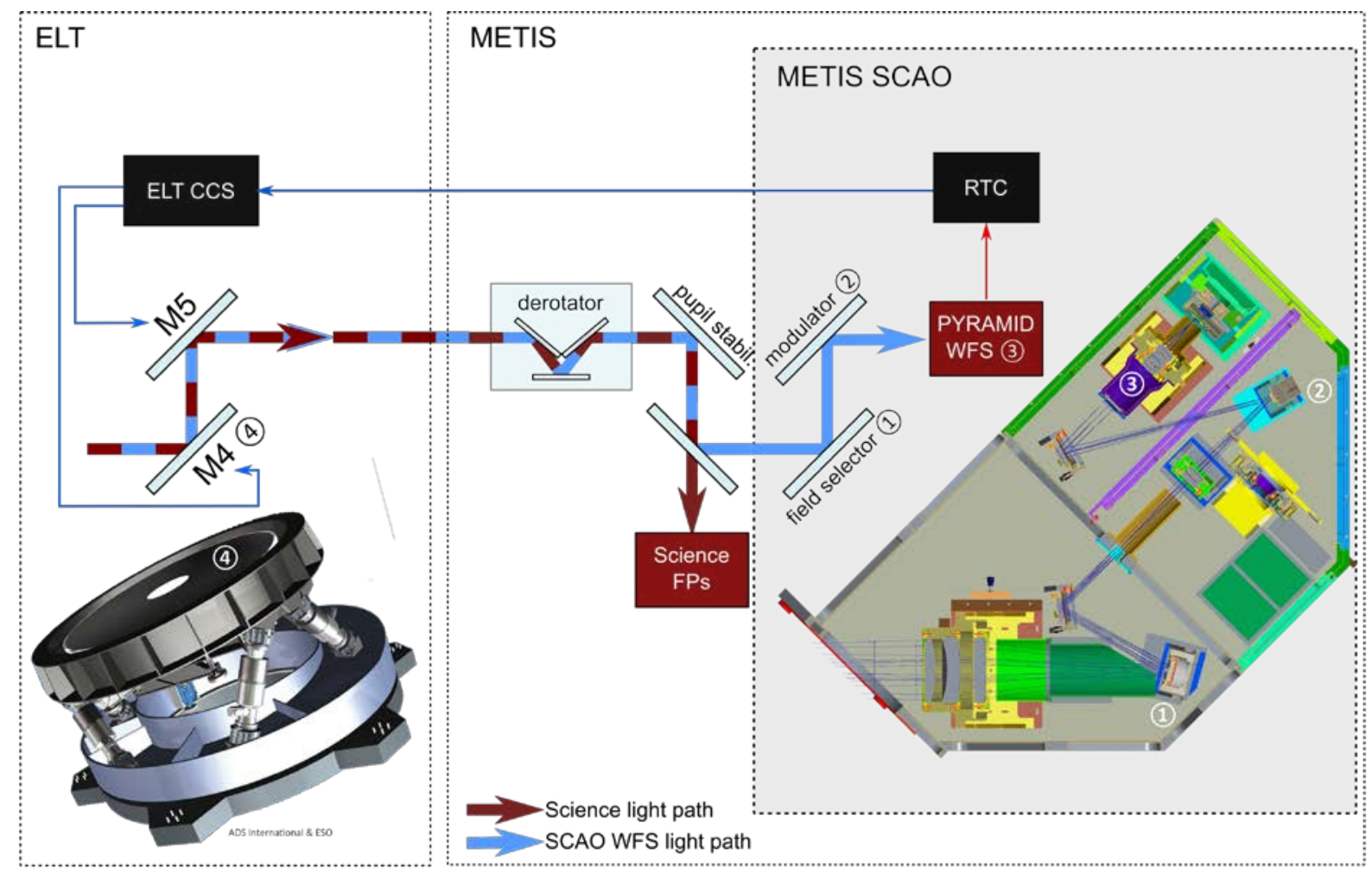

Figure 6 Control scheme for METIS SCAO. The light propagates through telescope and instrument. A pickoff mirror is used to direct the NIR part of the light (blue) to the SCAO module, which provides a field selector, a modulator, and a pyramid wavefront sensor to detect wavefront aberrations. The wavefront is reconstructed, and the correction signals are calculated by the RTC, which are being sent by the Central Control System to the actuators of the ELT's deformable mirror M4 and the field stabilization mirror M5.

\section{CORONAGRAPHY AND HIGH CONTRAST IMAGING}

A key feature of METIS is that it has been conceived to enable high-contrast imaging (HCI) and designed to reach the best possible performance in that observing mode. Imaging exoplanets and circumstellar emission around nearby stars is one of the key science cases of METIS, at a wavelength range that is generally considered as a sweet spot for such studies. METIS will feature two main HCI architectures, based on the apodizing phase plate (APP) and the ring-apodized vortex coronagraph (RAVC), respectively (Figure 7). These two architectures provide the optimum performance in terms of discovery space and sensitivity under different observational and environmental boundary conditions.

The expected high contrast imaging performance of METIS is illustrated in Figure 8. This performance estimate is based on end-to-end simulations, which include the influence of the SCAO correction, for the RAVC at L band. Figure 8 also shows the measured contrast curves from current HCI cameras on 8-m class telescopes for reference [21]. This comparison, however, must be taken with care, not only because it compares simulated to measured contrast curves, but also because our METIS simulations do not yet capture some potentially important contributors, such as non-common path aberrations (NCPA) and atmospheric dispersion. More details on the METIS HCI modes can be found in [22]. 


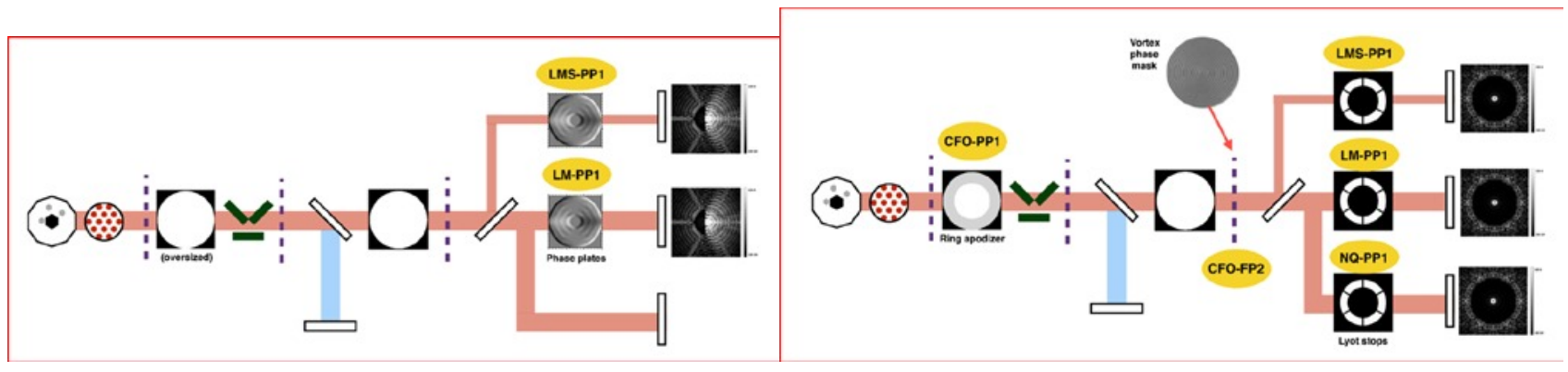

Figure 7 Simplified diagrams of the optical system, showing the locations of the coronagraphic masks at the various pupil planes (PP) and focal planes (FP) within METIS. Left: The apodized phase plates (APP). Right: The ring-apodized vortex coronagraph (RAVC).

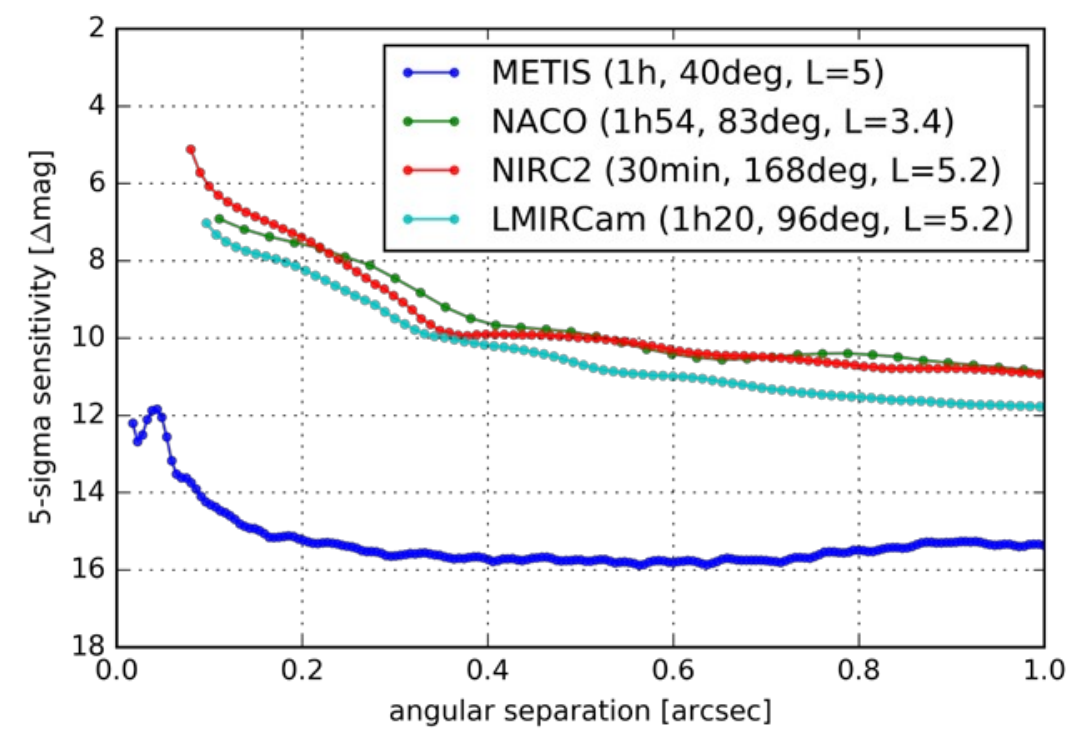

Figure 8 Simulated contrast curve for the METIS ring-apodized vortex coronagraph at $L$ band after 1 hour of onsky integration, assuming 40 degrees of sky rotation and no non-common path aberrations between the wavefront sensor camera and the science camera in the focal plane. Comparison curves from current $8 \mathrm{~m}$ class telescopes are shown as blue, green and red curves.

\section{WARM CALIBRATION UNIT}

METIS is equipped with a Warm Calibration Unit (WCU), located on top of the cryostat. The WCU delivers diagnostic tools for analyzing the image quality of METIS, its flux sensitivity, transmission stability and spectral response, both during the AIV phase and during regular operation at the ELT. It will also serve as the primary tool for internal alignment checks. The WCU is being designed to ensure the optimal characterization and calibration of METIS to deliver high-quality science-grade data, and to monitor the instrument response over its lifetime. More specifically, the WCU will be used to determine pixel-to-pixel detector characteristics, the spatial point-spread-function (PSF) across the field of view of the imagers, determine the spectral PSF of the spectrometers, and to provide the initial spectral calibration of the spectrometers. In addition, considering that METIS will be one of the three first scientific instruments at the ELT, the presence of a well-adapted troubleshooting system will help to better understand the interplay between the instrument and the telescope.

The WCU is installed on top of the main cryostat via a hexapod interface and can be operated independently from the other METIS subsystems. The light from the WCU is fed into METIS via a periscopic arm situated in front of the main 
entrance window. Several infrared light sources will be offered: a broadband thermal blackbody source of tunable and precisely controlled temperature, a set of tunable quantum cascade lasers (QCL), a tunable $\mathrm{CO}_{2}$ laser and a $3.39 \mu \mathrm{m}$ $\mathrm{HeNe}$ laser. All the light sources can produce uniformly illuminated at fields, single diffraction limited point sources, or combinations or grids of multiple diffraction-limited point sources in the focal planes of METIS. Figure 9 shows an illustration of the WCU with all major elements rendered in. More details on its functionalities and operating rationale can be found in [23], while a detailed description of its optical concept is given in [24].

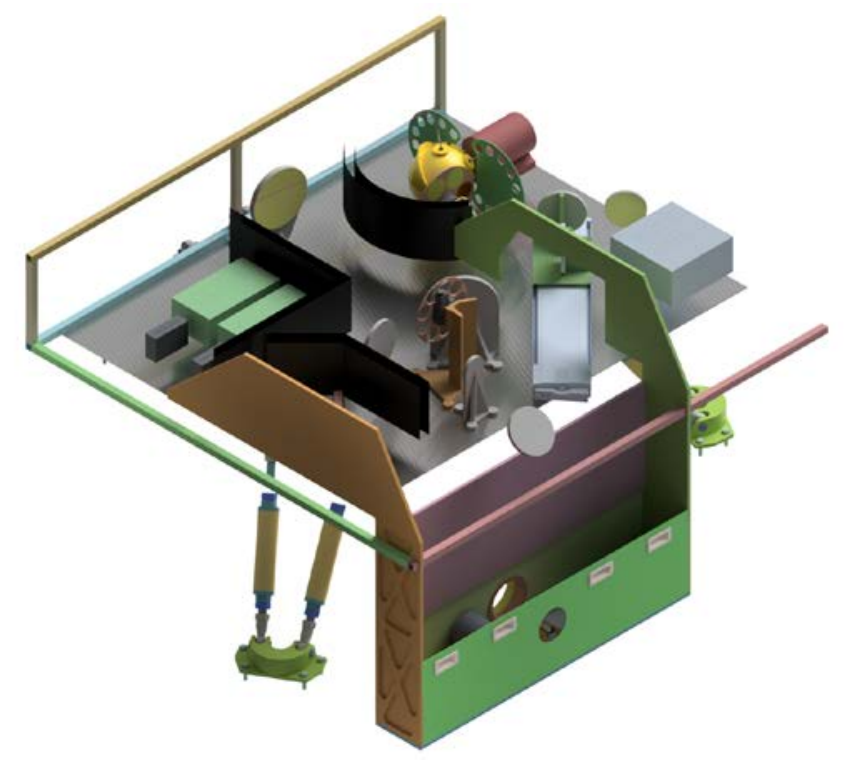

Figure 9 The warm calibration unit located on top of the main cryostat via a hexapod interface.

\section{OBSERVING MODES AND THEIR CALIBRATION}

\subsection{The main METIS observing modes}

At the most basic level METIS consists of two scientific sub-systems: the imager and the L/M high-resolution integral field spectrograph (LMS). The resulting observing modes, shown in Figure 10, reflect this architecture.

The imager can perform plain imaging through a set of broad- and narrow-band filters in each of its two arms. Observations in the N/Q camera will employ chopping, while chopping is optional for observations with the L/M camera. Nodding/dithering will likely be employed in both cases. Each imager arm has one or more grisms to enable longslit spectroscopy. The L/M arm has two grisms to disperse the entire atmospheric L-band or M-band onto the detector in one exposure, at a spectral resolution of $R_{\mathrm{L}} \sim 1500$ and $R_{\mathrm{M}} \sim 2000$, respectively. Equivalently, the N/Q arm has a grism with which the entire N-band spectrum can be recorded at $R_{\mathrm{N}} \sim 440$.

The LMS nominally observes in $40-80 \mathrm{~nm}$ wide passbands at a spectral resolution of $R=100,000$ at $4.65 \mu \mathrm{m}$, with a rectangular field covering $1.0^{\prime \prime} \times 0.5^{\prime \prime}$ in the along- $\times$ across-slice directions. The sampling of the focal plane at the image slicer is 0.5 (along) and 1.0 (across) times the FWHM of the diffraction-limited PSF at $3.0 \mu \mathrm{m}$. The different wavelength ranges can be selected using appropriate settings of the pre-dispersion prism and main dispersion grating angles.

A mode which provides extended wavelength coverage will also be available, allowing to instantaneously observe a 5 times wider spectral range at the expense of spatial coverage by the IFU. (In this mode only $\sim 1 / 5^{\text {th }}$ of the nominal spatial coverage by the IFU can be observed). The LMS pick-off is a grey beam splitter, sending $\sim 90 \%$ of the light to the spectrograph and letting the rest pass through to the imager. It is foreseen that LMS observations are always accompanied by parallel L/M imaging to allow for accurate astrometric registration of the LMS data.

METIS will also offer coronagraphic observations in most of the basic modes described above (see section 7 for details). The possibility of performing observations with Sparse Aperture Masking (SAM) by including appropriate pupil plane masks in the IMG is being investigated. Figure 10 gives an overview of the observing modes of METIS. 


\begin{tabular}{|c|c|c|c|}
\hline Mode & Band & Configuration & Science Driver \\
\hline \multirow{7}{*}{ 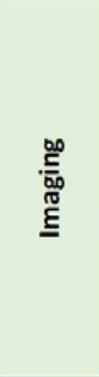 } & \multirow{4}{*}{ L/M } & direct & circum-stellar (YSOs) and circum-nuclear (AGN) structures \\
\hline & & APP & \multirow{3}{*}{ exoplanets (detection + characterization) } \\
\hline & & RAVC & \\
\hline & & SAM & \\
\hline & \multirow{3}{*}{$\mathrm{N} / \mathrm{Q}$} & direct & circum-stellar (YSOs) and circum-nuclear (AGN) structures \\
\hline & & RAVC & \multirow{2}{*}{ exoplanets (detection + characterization) } \\
\hline & & SAM & \\
\hline \multirow{2}{*}{ 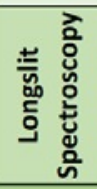 } & $\mathrm{L} / \mathrm{M}$ & entire band & ices in comets and star forming regions \\
\hline & $\mathbf{N}$ & entire band & $\begin{array}{l}\text { physics of circum-stellar and circum-nuclear } \\
\text { environments; sold-state chemistry \& mineralogy }\end{array}$ \\
\hline \multirow{4}{*}{ 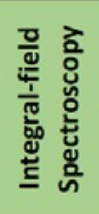 } & \multirow{2}{*}{ 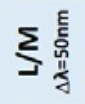 } & direct & kinematics and chemistry of circum-stellar environments \\
\hline & & RAVC (APP) & exoplanetary atmospheres \\
\hline & \multirow{2}{*}{ 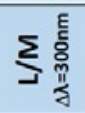 } & direct & chemical studies of the interstellar medium \\
\hline & & RAVC (APP) & exoplanetary atmospheres \\
\hline \multirow{2}{*}{ हิ } & L & \multirow{2}{*}{$\begin{array}{l}\text { diagnostic } \\
\text { mode }\end{array}$} & \multirow{2}{*}{ N/A } \\
\hline & $\mathrm{N}$ & & \\
\hline
\end{tabular}

Figure 10 The main METIS observing modes in the areas of imaging, longslit spectroscopy and integral field spectroscopy. Pupil imaging is shaded since it serves as a diagnostic mode, rather than a scientific observing mode. LMS observations are usually being done in parallel with $\mathrm{L} / \mathrm{M}$ imaging.

\subsection{Thermal background subtraction}

One of the prime challenges for METIS to reach its nominal sensitivity is the accurate suppression, measurement, and removal of the thermal emission from the telescope and the sky without biasing the scientific image. This is already challenging for current thermal-IR imagers on $8 \mathrm{~m}$-class telescopes, but will be aggravated for METIS by having six warm telescope mirrors in front of the cryostat entrance window. A preview of this challenge is shown in Figure 11 which compares the "chop-difference" images from VLT/VISIR (two warm reflections upstream) with the corresponding ones from VLTI/MIDI (some 20 warm reflections upstream).

An additional complication for the calibration of METIS arises from the fact that we cannot use the secondary mirror of the ELT for chopping at several $\mathrm{Hz}$, but need to use an instrument-internal cryo-chopper [25]. Switching between onand off-axis positions will thus cause the footprint of the beam to vary on all mirrors upstream of the cryo-chopper. This wander may produce a significant residual in the difference signal. We are therefore exploring strategies how to reach the shot noise limit of the thermal background despite these effects. Since we cannot avoid these residuals, we are trying to quantitatively understand their origin in existing systems, and build a model of these systematics for their removal within the data reduction pipeline.

Based on new observations with VISIR, we are trying to find out which warm elements in the VLT pupil are responsible for the structures that we see (Figure 11, left). The main likely culprits are the warm support cell for the M3 mirror and the spiders of the secondary mirror. In parallel we are attempting to build a thermal straylight model for the full VLT+VISIR system (Figure 11, right) to quantitatively assess whether the assumed components could produce the observed residual structures.

We are also exploring new methods for the background subtraction and in particular drift- or slow-scanning techniques [26] in order to calibrate detector-gain fluctuations and other effects which contribute noise power in the $1-10 \mathrm{~Hz}$ range, without the need for chopping. 

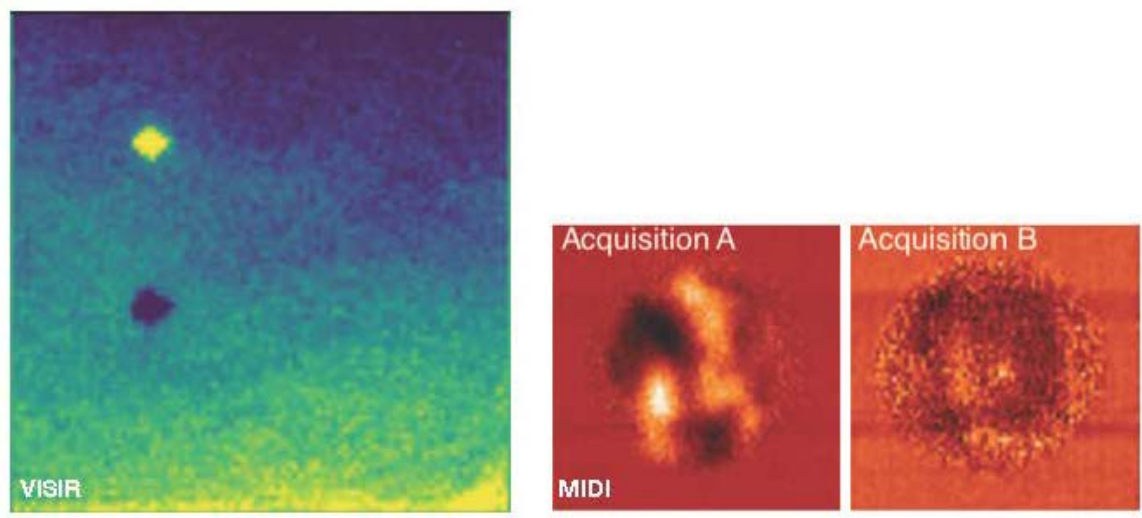

Figure 11 Images after chop-reduction from VLT/VISIR (left) and VLTI/MIDI (right). In the VISIR chopdifference frame, the background can be described as a gradient with additional higher-order terms. In the MIDI chop-difference frames, the residuals are very time- and telescope-dependent. Here the level of the residuals is much stronger, and in some frames ("Acquisition A") the residual image shows structure well above the nominal sensitivity limit.
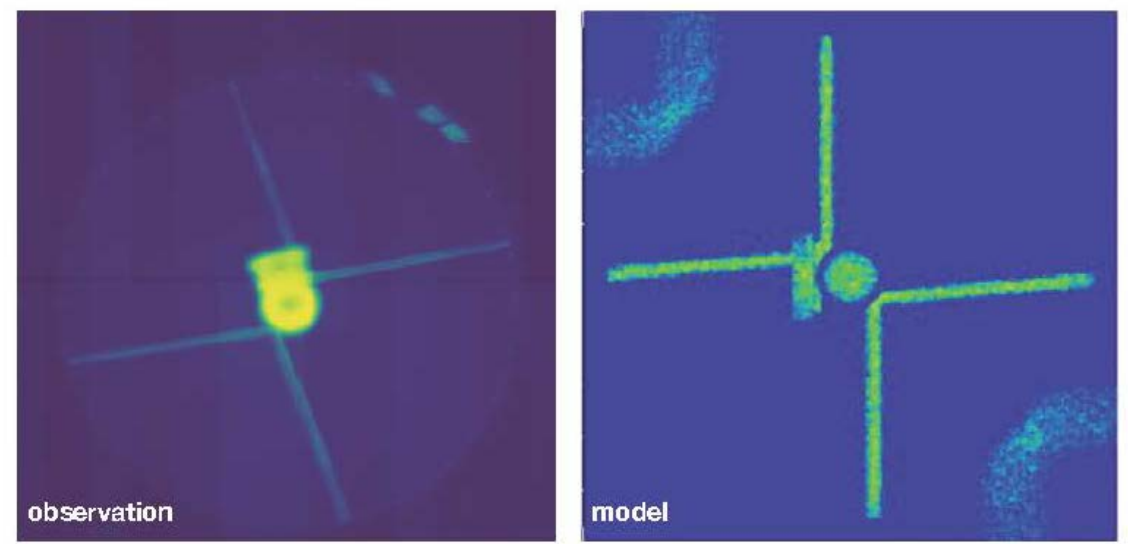

Figure 12 Left: The warm elements in the VLT pupil, observed at $10 \mu \mathrm{m}$ with VISIR. Right: Thermal model of the same configuration.

\subsection{Wavelength and telluric calibration}

The basic wavelength calibration can be performed with the WCU (section 8). In addition to this, we will use the telluric absorption and emission lines of the atmosphere, which are imprinted on each science spectrum, for wavelength calibration. This forest of narrow absorption lines will provide an optimal wavelength calibration for any source position within the slit, although they may not have the required signal-to-noise ratio for fainter sources.

The observation of the telluric absorption spectrum, in combination with a radiative transfer model, such as molecfit [27], [28], will further allow us to perform the telluric calibration without the observation of a telluric calibrator star, as has been the standard calibration technique in the thermal IR so far. While this modelling technique has been shown to work very well with high spectral resolution data in the optical and near infrared, we are currently exploring the limitations of this technique in the thermal IR, and for low-to-medium spectral resolutions as provided by our long-slit spectrographs. 


\section{SCHEDULE}

Last but not least, the schedule (Figure 13) is an important driver for the development of METIS. The agreement on the design and construction of METIS with ESO has been signed on September 28th, 2015. At this point in time, METIS is in the Preliminary Design Phase. The Preliminary Design Review (PDR) is set in early May 2019. It is followed by the Final Design phase which covers a period of two years (Final Design Review, FDR, in 2021), after which manufacturing starts. A pre-final design review is foreseen to happen about six months before FDR, to allow for an earlier ordering of the long-lead items, such as optics, cryo-mechanisms and raw material.

The Assembly, Integration and Verification (AIV) is divided in two phases, a sub-system phase (named Phase \#1) and a system phase (Phase \#2). In the system phase the entire instrument is assembled and integrated, tested and verified at the system AIV facility in the Netherlands. In practice, sub-system and system AIV do overlap as sub-systems will arrive according to plan at the system AIV facility.

The Preliminary Acceptance in Europe (PAE) marks the readiness of the instrument, after which the transport to Chile kicks off. This is planned for 2024. After integration and verification at the observatory site in 2025 , the instrument will be commissioned on the ELT. The commissioning is foreseen to be completed in 2026, 10.5 years after kick off.

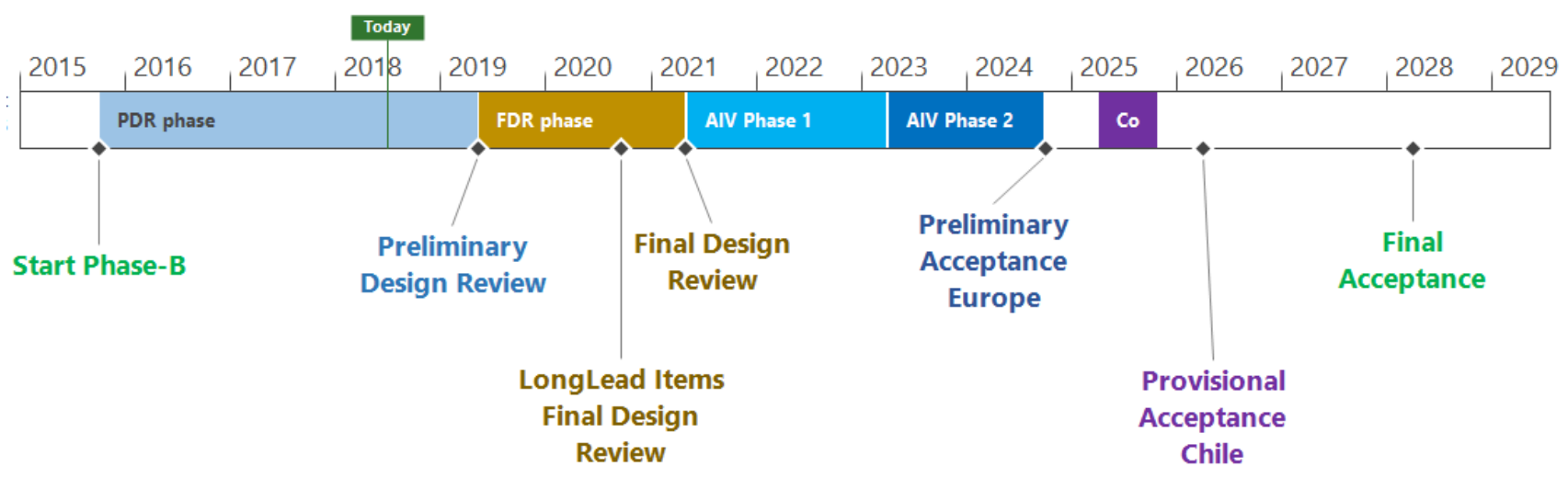

Figure 13 The METIS milestone schedule as of July 2018.

\section{REFERENCES}

[1] Brandl, Bernhard R. et al., 'METIS: the mid-infrared E-ELT imager and spectrograph', Proceedings of the SPIE, Volume 9147, 91472118 pp. (2014)

[2] Brandl, Bernhard R. et al., 'Status of the mid-infrared E-ELT imager and spectrograph METIS', Proceedings of the SPIE, Volume 9908, 99082015 pp. (2016)

[3] Pinilla, P., Birnstiel, T., Ricci, L., Dullemond, C. P., Uribe, A. L., Testi, L., Natta, 'Trapping dust particles in the outer regions of protoplanetary disks', Astronomy \& Astrophysics, Volume 538, A114 (2012)

[4] Ataiee, S., Pinilla, P., Zsom, A., Dullemond, C. P., Dominik, C., Ghanbari, 'Asymmetric transition disks: Vorticity or eccentricity?', Astronomy \& Astrophysics, Volume 553, L3 (2013)

[5] de Juan Ovelar, M., Min, M., Dominik, C., Thalmann, C., Pinilla, P., Benisty, M., Birnstiel, 'Imaging diagnostics for transitional discs', Astronomy \& Astrophysics, Volume 560, A111 (2013)

[6] Taha, A. S., Labadie, L., Pantin, E., Matter, A., Alvarez, C., Esquej, P., Grellmann, R., Rebolo, R., Telesco, C., Wolf, S., 'The spatial extent of polycyclic aromatic hydrocarbons emission in the Herbig star HD 179218', Astronomy \& Astrophysics, Volume 612, A15 (2018)

[7] Snellen, I., de Kok, R., Birkby, J. L., Brandl, B., Brogi, M., Keller, C., Kenworthy, M., Schwarz, H., Stuik, R., 'Combining high-dispersion spectroscopy with high contrast imaging: Probing rocky planets around our nearest neighbors', Astronomy \& Astrophysics, Volume 576, A59 (2015) 
[8] Snellen, Ignas A. G., Brandl, Bernhard R., de Kok, Remco J., Brogi, Matteo, Birkby, Jayne, Schwarz, Henriette, 'Fast spin of the young extrasolar planet $\beta$ Pictoris b', Nature, Volume 509, Issue 7498, pp. 63-65 (2014)

[9] Bryan, Marta L., Benneke, Björn, Knutson, Heather A., Batygin, Konstantin, Bowler, Brendan P., 'Constraints on the spin evolution of young planetary-mass companions', Nature Astronomy, Volume 2, p. 138-144 (2018)

[10] Crossfield, Ian J. M., 'Doppler imaging of exoplanets and brown dwarfs', Astronomy \& Astrophysics, Volume 566, A130 (2014)

[11] Molliere \& Snellen, (2018 in prep)

[12] Quanz, Sascha P., Crossfield, Ian, Meyer, Michael R., Schmalzl, Eva, Held, Jenny, 'Direct detection of exoplanets in the 3-10 $\mu \mathrm{m}$ range with E-ELT/METIS', International Journal of Astrobiology, Volume 14, Issue 2, pp. 279-289 (2015)

[13] Sozzetti, Alessandro, Damasso, Mario, 'The gaia survey contribution to EChO target selection and characterization', Experimental Astronomy, Volume 40, Issue 2-3, pp. 595-600 (2015)

[14] http://www.exoplanet.eu

[15] Agócs, T. et al., 'Preliminary optical design for the common fore optics of METIS,' Proc. SPIE 9908, Paper 99089Q (2016).

[16] Straubmeier, C. et al., 'The Warm Calibration Unit of METIS optical design and principle of operation,' Proc. SPIE 10702, Paper 10702-318 (2018).

[17] Agócs, T. et al., 'End to end optical design and wavefront error simulation of METIS,' Proc. SPIE 10702, Paper 10702-353 (2018).

[18] Agócs, T., et al., "Optical tests of the Si immersed grating demonstrator for METIS," Proc. SPIE 9912-41 (2016).

[19] Wells, M., et al., "Spectral slicing for METIS: an efficient alternative to cross-dispersion," Proc SPIE 9912-202 (2016).

[20] Bertram, T., Absil, O., Bizenberger, B., Briegel, F., Cantalloube, F. Carlomagno, B. , Cárdenas Vázquez, M. C., Feldt, M., Glauser, A. M., Henning, T., Hippler, S., Huber, A., Hurtado, N., Kenworthy, M. A., Kulas, M., Mohr, L., Naranjo, V., Neureuther, P., Obereder, A., Rohloff, R.-R., Scheithauer, S., Shatokhina, I, Stuik, R., and van Boekel, R. "Single Conjugate Adaptive Optics for METIS", SPIE 10703, 10703-41 (2018)

[21] Absil, Olivier, Mawet, Dimitri, Karlsson, Mikael, Carlomagno, Brunella, Christiaens, Valentin, Defrère, Denis, et al., 'Three years of harvest with the vector vortex coronagraph in the thermal infrared', Proceedings of the SPIE, Volume 9908, 99080Q 14 pp. (2016)

[22] Matthew A. Kenworthy, Olivier Absil, Brunella Carlomagno, Tibor Agócs, Emiel H. Por, Bernhard Brandl, Frans Snik, 'A review of high contrast imaging modes for METIS', Proc. SPIE 10702, Paper 10702-369 (2018)

[23] Nicola Baccichet, Lucas Labadie, Steffen Rost, Christian Straubmeier, Michael Wiest, Leonard Burtscher, Tibor Agócs, Willem Jellema, Ronald Roelfsema, Roy van Boekel, Adrian Glauser, Bernhard Brandl, Felix Bettonvil, Jeffrey Lynn, Andreas Eckart, 'The calibration unit of the mid-infrared E-ELT instrument METIS', Proc. SPIE 10702, Paper 10702-330 (2018)

[24] Christian Straubmeier, Lucas Labadie, Nicola Baccichet, Michael Wiest, Andreas Eckart, 'The warm calibration unit of METIS: optical design and principle of operation', Proc. SPIE 10702, Paper 10702-318 (2018).

[25] Paalvast, S., Huisman, R., Brandl, B., Janssen, H., Jayawardhana, B., Molster, F., Teuwen, M., Venema, L., "Development and characterization of a 2D precision cryogenic chopper for METIS", Proc. SPIE 9151 (2014).

[26] Ohsawa, Ryou, Sako, Shigeyuki, Miyata, Takashi, Kamizuka, Takafumi, Okada, Kazushi, Mori, Kiyoshi, Uchiyama, Masahito S., Yamaguchi, Junpei, Fujiyoshi, Takuya, Morii, Mikio, Ikeda, Shiro, '"Slow-scanning" in Ground-based Mid-infrared Observations', Astrophysical Journal, Volume 857, Issue 1, article 37 (2018)

[27] Smette, A., Sana, H., Noll, S., Horst, H., Kausch, W., Kimeswenger, S., Barden, M., Szyszka, C., Jones, A. M., Gallenne, A., Vinther, J., Ballester, P., Taylor, J. A., 'Molecfit: A general tool for telluric absorption correction. I. Method and application to ESO instruments', Astronomy \& Astrophysics, Volume 576, A77 (2015)

[28] Kausch, W., Noll, S., Smette, A., Kimeswenger, S., Barden, M., Szyszka, C., Jones, A. M., Sana, H., Horst, H., Kerber, F., 'Molecfit: A general tool for telluric absorption correction. II. Quantitative evaluation on ESOVLT/X-Shooterspectra', Astronomy \& Astrophysics, Volume 576, A78 (2015) 